\title{
Occurrence of Second Mesio-buccal Canal in Maxillary First Permanent Molar: A CBCT Studụ
}

\author{
Dr Sandhya Shrestha,' Dr Manish Raj Sapkota, ${ }^{2}$ r Ankit Saha, ${ }^{3}$ Dr Santosh Rajbhandari, ${ }^{4}$ Dr Pratyush Sapkota \\ ${ }^{14}$ Asst Prof, ${ }^{5}$ Lecturer, ${ }^{2}$ Dental Surgeon, Dept of Conservative Dentistry \& Endodontics \\ 3Lecturer, Dept of Oral Medicine \& Radiology \\ Kantipur Dental College, Kathmandu, Nepal
}

Correspondence: Dr Sandhya Shrestha; Email: sandhyaendo@gmail.com

\section{ABSTRACT}

Introduction: Complexity in root canal morphology leads to chances of failure in endodontic treatment due to missed canal. Availability of Cone-beam Computed Tomography (CBCT) has contributed to assess second mesiobuccal canals in maxillary first molars.

Objective: To study the presence of second mesiobuccal (MB2) canals, classify morphology of canals according to Vertucci's classification and to measure the distance between MBI and MB2 canals.

Materials \& Method: 72 CBCT scans including 115 permanent maxillary first molars were evaluated by endodontist and oral radiologist separately using Dicom software to study the mesiobuccal root canal morphology. Oral radiologist and endodontist individually evaluated the scans through coronal, axial and oblique views to study canal configurations. Vertucci's Classification was used to classify the canal configurations. Statistical tests were done to assess the gender variation and arch side difference.

Result: Among 115 images evaluated; 30.4\% samples had second mesiobuccal canal. The most common canal configuration was Type I (60.9 \%) in mesiobuccal root. The mean distance between MB1 and MB2 canals was $2.39 \mathrm{~mm}$ in female and 2.56 $\mathrm{mm}$ in male.

Conclusion: The occurrence of second canal in mesiobuccal root of maxillary permanent molar is considerable in Nepali patients. CBCT can be an effective tool to detect MB2 canal and other complexities of root canal system to achieve clinical success.

Keywords: $\mathrm{CBCT}$, endodontic, mesio-buccal canal, Vertucci's classification

\section{INTRODUCTION}

Maxillary first molar is one of the longest standing teeth in the dental arch which serves as a key of occlusion' and anchorage in most of the orthodontic treatments. However, they are susceptible to various insults such as dental caries, attrition etc. The tooth is very important in masticatory process and overall balance of the occlusion; hence it is strategically important to maintain the tooth in dental arch as far as possible, which often requires endodontic treatment.

Success of endodontic treatment is attributed to adequate cleaning, disinfecting, shaping, and three dimensionally sealing the root canal system. ${ }^{2}$ The process is routinely challenged by the complexity of root canal morphology. Knowledge of root canal morphology is a key factor which aids in successful treatment outcome. Maxillary molars possess complex morphology which has highest rate of clinical failures due to missed second mesio-buccal canal with substantial variations in mesiobuccal root morphology. ${ }^{3}$

Numerous studies have been conducted to investigate root canal configurations of maxillary first molars adopting both in vitro and in vivo methods such as canal staining and clearing techniques, contrast medium-enhanced radiography, micro-computed tomographic imaging, intraoral periapical radiographs, dentin troughing under magnification, clinical operating microscope, ultrasonics and cone-beam computed tomographic (CBCT) imaging methods. ${ }^{4}$ Among all, later five have been utilized in clinical practice and $\mathrm{CBCT}$ is considered as gold standard. $\mathrm{CBCT}$ is helpful for clinicians in identifying all canals and their configurations in clinical scenario. ${ }^{5}$

This study aims to assess the presence of second mesiobuccal canal in mesiobuccal root, classify the canals according to Vertucci and measure distance between first and second mesiobuccal canals utilizing CBCT images. 

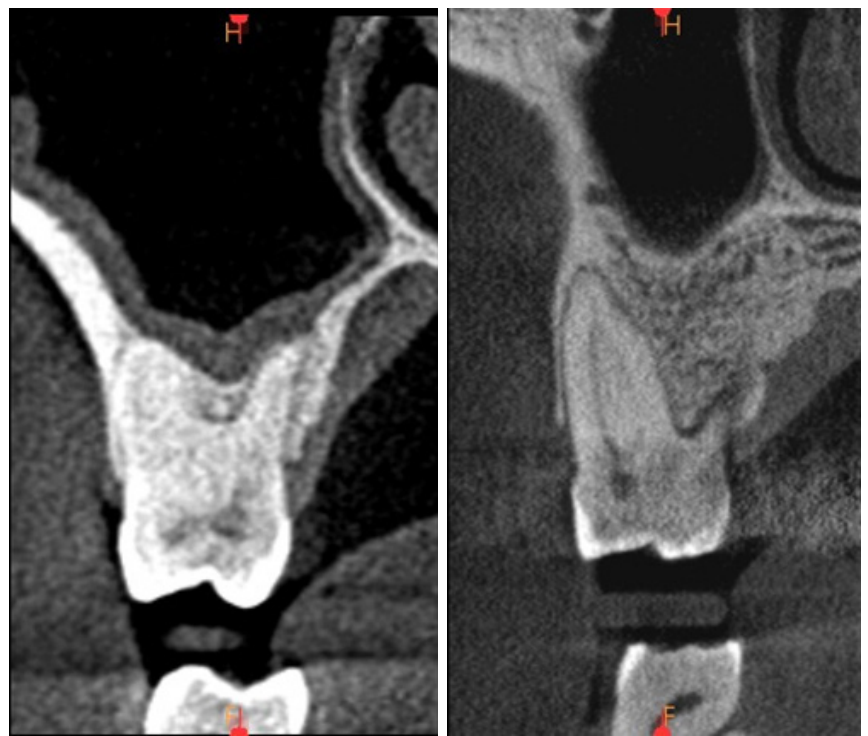

Figure 1: CBCT scans

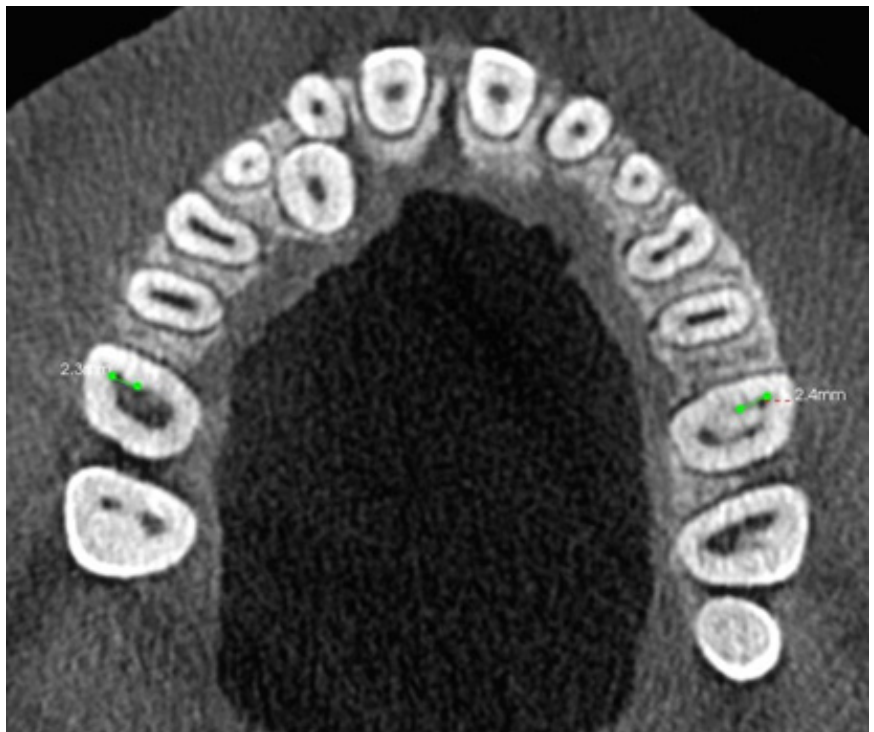

The data were anonymized before evaluation. The images were evaluated by using Dicom CS 3D imaging software version 3.5.18. To standardize the data a voxel size of $180 \mu$ was chosen across all scans. An oral radiologist and endodontist individually evaluated the scans through coronal, axial and oblique views to check the morphology of canals (Figure 1), assess the presence of MB2 canal, classify canals based on Vertucci's Classification (Figure 2), and measure the distance between MB1 and MB2 canals if present at the level of pulpal floor.

The data was recorded in excel sheet and was statistically analyzed using SPSS 20 software. Statistical tests were done by using chi square test to check the association between M2 canal and gender and arch side. Independent t-test was used to compare the statistical difference between distance between the canals and gender. Level of significance was set at $p<0.05$.

Kappa test was done to check the inter-observer reliability between the oral radiologist and endodontist. Cronbach alpha test was done to check the inter-observer reliability in measuring the distance between $M B 1$ and MB2 canals. restoration, root resorption or gross loss of coronal tooth structure. Blurred or unclear images were also excluded.

\section{Vertucci's Classification of root canal morphology}

\begin{tabular}{|c|l|c|}
\hline Type & \multicolumn{1}{|c|}{ Description } & Configuration \\
\hline Type I & A single canal from pulp chamber to the canal terminus & $1-1$ \\
\hline Type II & $\begin{array}{l}\text { Two separate canals leaving the chamber but merging short of the canal terminus to form a single } \\
\text { canal }\end{array}$ & $2-1$ \\
\hline Type III & A single canal that divides into two and subsequently merges to exit as one & $1-2-1$ \\
\hline Type IV & Two distinct canals from pulp chamber to the canal terminus & $2-2$ \\
\hline Type V & A single canal leaving the chamber and dividing into two separate canals at the canal terminus & $1-2$ \\
\hline Type VI & $\begin{array}{l}\text { Two separate canals leaving the pulp chamber, merging in the body of the root, and dividing } \\
\text { again into two distinct canals short of the canal terminus }\end{array}$ & $2-1-2$ \\
\hline Type VII & A single canal that divides, merges and exits into two distinct canals short of the canal terminus & $1-2-1-2$ \\
\hline Type VIII & Three distinct canals from pulp chamber to the canal terminus & $3-3$ \\
\hline
\end{tabular}


Type I

(1-1)

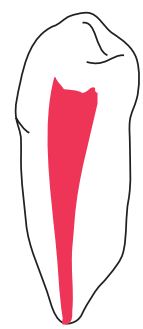

Type II

(2-1)

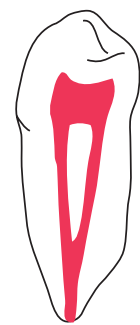

Type III

(1-2-1)

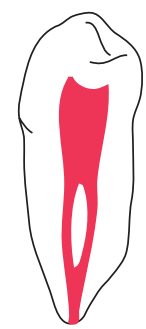

Type IV

(2-2)

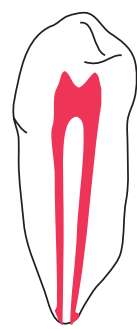

Type $V$

(1-2)

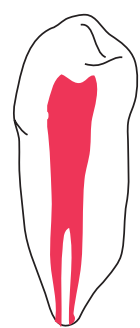

Type VI

(2-1-2)

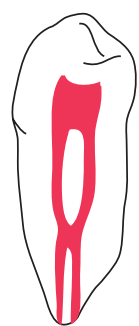

TypeVII

(1-2-1-2)

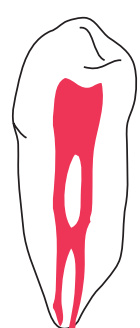

Type VIII

(3-3)

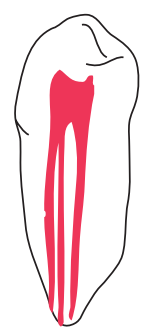

Figure 2: Diagrammatic representation of Vertucci's classification

\section{RESULT}

A total of 115 maxillary first molar samples on 72 CBCT scans were observed. Among all, 53.9\% were right and $46.1 \%$ were left sided first molar. The samples comprised of $55.56 \%$ male and $44.44 \%$ female subjects. Mean age of the sample was 33.53 years (SD 14.60) with the age range 16-60 years.

The study revealed that $30.4 \%$ of maxillary first molars showed the presence of MB2 canal in mesiobuccal root. The most common canal configuration was Vertucci's Type I (60.9\%), followed by Type II (19.1\%), Type IV (7.8\%) (Graph 1). There was no finding of Type $V$ and Type $\mathrm{VI}$ in any sample.

The mean distance between mesiobuccal-1 (MB1) and mesiobuccal-2 (MB2) canals were $2.56 \mathrm{~mm}$ in male and $2.39 \mathrm{~mm}$ in female. There was no significant difference between distance between the canals and gender $(p=0.506)$ (Table 1).
There was no statistically significant association between the presence or absence of MB2 canal and the gender $(p=0.138$; Chi Square $=2.199)$ as well as sides $(p=0.724$; Chi Square $=0.125)($ Table 2,3)

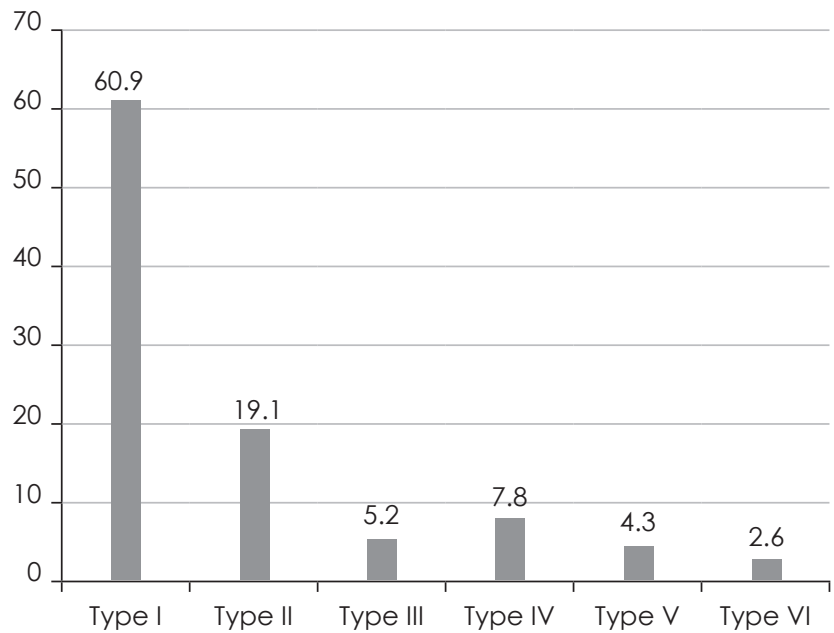

Graph 1: Frequency of types of canal configuration (\%)

Table 1: t-Test of significance for comparison of distance between canals Vs gender

\begin{tabular}{|l|c|c|c|c|}
\hline & Mean Distance $\mathbf{( m m})$ & SD & t-Value & p-Value \\
\hline Female & 2.39 & 0.75 & -0.673 & 0.506 (NS) \\
\hline Male & 2.56 & 0.65 & & \\
\hline
\end{tabular}

Table 2: Association between M2 canal and gender

\begin{tabular}{|c|c|c|c|c|c|}
\hline \multirow{2}{*}{ MB2 Canal } & \multicolumn{2}{|c|}{ Gender } & \multirow{2}{*}{ Total } & \multirow{2}{*}{$x^{2}$} & \multirow{2}{*}{$p$-Value } \\
\hline & Female & Male & & & \\
\hline \multirow{2}{*}{ Present } & 11 & 24 & 35 & \multirow{5}{*}{2.199} & \multirow{5}{*}{0.138 (NS) } \\
\hline & $22.9 \%$ & $35.8 \%$ & $30.4 \%$ & & \\
\hline \multirow{2}{*}{ Absent } & 37 & 43 & 80 & & \\
\hline & $77.1 \%$ & $64.2 \%$ & $69.6 \%$ & & \\
\hline Total & 48 & 67 & 115 & & \\
\hline
\end{tabular}

Table 3: Association between M2 canal and side

\begin{tabular}{|c|c|c|c|c|c|}
\hline \multirow{2}{*}{ MB2 Canal } & \multicolumn{2}{|c|}{ Maxillary First Molar } & \multirow{2}{*}{ Total } & \multirow{2}{*}{$X^{2}$} & \multirow{2}{*}{$p$-Value } \\
\hline & Right & Left & & & \\
\hline \multirow{2}{*}{ Present } & 18 & 17 & 35 & \multirow{5}{*}{0.125} & \multirow{5}{*}{0.724 (NS) } \\
\hline & $29.6 \%$ & $32.1 \%$ & $30.4 \%$ & & \\
\hline \multirow{2}{*}{ Absent } & 44 & 36 & 80 & & \\
\hline & $67.9 \%$ & $69.6 \%$ & $71.0 \%$ & & \\
\hline Total & 62 & 53 & 115 & & \\
\hline
\end{tabular}


Inter-observer agreement was high for the presence or absence of MB2 canal (Kappa $=0.918, \mathrm{p}<0.001)$, canal configuration (Kappa $=0.876, \mathrm{p}<0.001$ ) and distance between MB1 and MB2 (Chronbach's alpha $=0.976)$.

\section{DISCUSSION}

Morphological complexity in maxillary first molar often results in failure to identify and locate second mesiobuccal canal leading to endodontic treatment failure. ${ }^{6-8}$

Various studies have reported various occurrences of MB2 canal in maxillary permanent first molar. Hartwell et al reported 40-95\%, ${ }^{2}$ Weine et al reported $51 \%$ prevalence, ${ }^{9}$ Buhrley et al reported $93 \%,{ }^{10}$ Pardo et al $23.81 \% .^{11}$ The present study revealed $30.4 \%$ occurrence of MB2 canal which is consistent with the study of Hartmann et al with $29.2 \% .^{12}$ The abridgement of literature on prevalence of MB2 in maxillary molar is enlisted in Table $4 .^{11}$

The variation in results in different studies is attributed to difference in age, gender, ethnicity/race, procedures and conditions of conducting research. ${ }^{13}$ Alacam et al compared direct observation, microscope, combination of microscope and ultrasonic method and dissecting techniques for evaluating MB2. The results were $62 \%, 67 \%, 74 \%$ and $82 \%$ respectively revealing difference in results. ${ }^{14}$ Pardo et al compared the efficacy of three methods of canal identification; viz $C B C T$, clinical analysis and operating microscope and revealed $\mathrm{CBCT}$ as the best in finding the canals. ${ }^{11}$

In the present study, canal configurations were identified according to Vertucci's classification system $^{15}$ revealing the most prevalent type as Type I in $60.9 \%$. However, Faramarzi et al found Type || canal configuration as the most common in $71.3 \% .^{16}$ According to Rwenyonyi et al; Type I was the most frequent accounting up to 75.1-100\%. ${ }^{17}$ Synopses of various literatures regarding canal configuration is listed in Table $5 .{ }^{18}$

MB2 canal orifice in pulpal floor of the mesiobuccal root is commonly situated within the developmental groove on an approximate line between MBI and the palatal orifice, parallel to mesial marginal ridge. ${ }^{7}$ The present study found maximum and minimum distance between MB1 and MB2 orifices as $3.80 \mathrm{~mm}$ and 1.40 $\mathrm{mm}$ respectively, with mean value being $2.39 \mathrm{~mm}$ and $2.56 \mathrm{~mm}$ in female and male respectively. These values are in accordance with Faramarzi et al with maximum and minimum distance as $4 \mathrm{~mm}$ and $1.5 \mathrm{~mm}$ respectively. ${ }^{16}$ Zhang et al ascertained that MB2 canal lies less than $1 \mathrm{~mm}$ mesially to MB-P line and $2 \mathrm{~mm}$ palatally to $M B$ orifice. ${ }^{19}$ Furthermore, no statistically significant association was found among gender groups and bilateral sides in occurrence of MB2 canal. These findings are is consistent with the study of Das et al. ${ }^{20}$

Table 4: Occurrence of MB2 canal in maxillary first molar in various studies

\begin{tabular}{|c|c|c|c|c|}
\hline Investigator & Year & $\mathbf{N}$ & Model & $\%$ \\
\hline Weine et al & 1969 & 208 & in vitro & 62 \\
\hline Pineda & 1973 & 245 & in vitro & 54.3 \\
\hline Hartwell \& Belizzi & 1982 & 1976 & in vitro & 18.6 \\
\hline Weller \& Hartwell & 1989 & 1134 & in vitro & 39.1 \\
\hline Kulild \& Peters & 1990 & 83 & in vitro & 95.2 \\
\hline Fogel et al & 1994 & 208 & in vivo & 71.2 \\
\hline Stropko & 1999 & 1732 & in vivo & 73.2 \\
\hline Sempira \& Hartwell & 2000 & 200 & in vivo & 33.1 \\
\hline Buhrley et al & 2002 & 660 & in vivo & 93 \\
\hline Baldassari-Cruz et al & 2002 & 39 & in vitro & 90 \\
\hline Coutinho Filho et al & 2006 & 108 & in vitro & 90.7 \\
\hline Smadi \& Khraisat & 2007 & 100 & in vitro & 77.32 \\
\hline Alaçam et al & 2008 & 100 & in vitro & 82 \\
\hline Hartmann et al & 2009 & 65 & in vitro & 29.2 \\
\hline Baratto Filho et al & 2009 & 100 & in vitro \& in vivo & 67.14 \\
\hline Blattner et al & 2010 & 20 & in vitro & 68.4 \\
\hline Pardo et al & 2012 & 42 & in vitro & 23.81 \\
\hline Shrestha et al & 2017 & 115 & in vitro & 30.4 \\
\hline
\end{tabular}


Table 5: Canal configuration in mesiobuccal root of maxillary first molar in various studies

\begin{tabular}{|l|c|c|c|l|c|c|c|c|}
\hline \multicolumn{1}{|c|}{ Investigator } & Year & N & Country & Study Technique & Type I & Type II & Type III & Type IV \\
\hline Weine & 1969 & 208 & USA & Vertical Sectioning & 48.5 & 37.5 & 14.0 & 0 \\
\hline Pineda & 1973 & 262 & Mexico & Radiographic & 39.3 & 12.2 & 35.7 & 12.8 \\
\hline Seldberg & 1973 & 100 & USA & Horizontal Sectioning & 38.0 & 37.0 & 25.0 & 0 \\
\hline Evenot & 1980 & 378 & France & Radiographic and microscopic & 28.8 & 23.5 & 38.8 & 8.8 \\
\hline Vertucci & 1984 & 100 & USA & Decalcified \& decayed & 45.0 & 37.0 & 18.0 & 0 \\
\hline Weine et al & 1996 & 300 & Japan & Files in extracted teeth & 42.0 & 24.2 & 30.4 & 3.4 \\
\hline Shrestha et al & 2017 & 115 & Nepal & CBCT & 60.9 & 19.1 & 5.2 & 7.8 \\
\hline
\end{tabular}

\section{CONCLUSION}

The occurrence of second canal in mesiobuccal root of maxillary permanent molar is considerable i.e. $30.4 \%$ in Nepali patients' sample. Clinicians should therefore assume the presence of MB2 canal during the endodontic procedure to optimize the treatment result. The clinicians should explore 1.4-3.8 $\mathrm{mm}$ distance palatally in an imaginary line joining MBI and palatal orifice to access MB2 canal orifice. Conventional radiographs usually do not yield the presence of MB2 canal; thus CBCT is the gold standard diagnostic tool in complex, doubtful and in retreatment of missed canal cases.

\section{OJN}

\section{REFERENCES}

1. Atkinson SR. A key to occlusion. Am J Orthod. 1968; 54(3):217-33.

2. Hartwell G, Appelstein CM, Lyons WW, Guzek ME. The incidence of four canals in maxillary first molars: A clinical determination. J Am Dent Assoc 2007; 138:1344-6.

3. Vertucci FJ, Haddix JE, Britto LR (2006) Tooth morphology and access cavity preparation. In: Cohen S, Hargreaves KM, ed. Pathways of the Pulp, 9th Ed. St. Louis: Mosby Elsevier, p. 203.

4. Zhang R, Yang H, YU X, Wang $\mathrm{H}$, HU T, Dummer PM. Use of CBCT to identify the morphology of maxillary permanent molar teeth in a Chinese subpopulation. Int Endod J.. 2011; 44(2):162-9.

5. Matherne RP, Angelopoulos C, Kulild JC, Tira D. Use of cone-beam computed tomography to identify root canal systems in vitro. J Endod. $2008 ; 34(1): 87-9$

6. Vertucci FJ. Root canal morphology and its relationship to endodontic procedures. Endod Topics. 2015; 10(1):3-29.

7. Torbay C, Nasseh I, Sokhn S. Use of CBCT in the detection of second mesiobuccal (MB2) canal in maxillary first molar. J Dent Health Oral Disord Therapy 2016; 4(6):134-7.

8. Vertucci Fj. Root canal anatomy of the human permanent teeth. Oral Surg Oral Med Oral Pathol.1984; 58(5):589-99

9. Weine FS, Healey HJ, Gerstein H, Evanson L. Canal configuration in the mesiobuccal root of the maxillary first molar and its endodontic significance. Oral Surg Oral Med Oral Pathol. 1969; 28(3):419-25

10. Buhrley LJ, Barrows MJ, BeGole EA, Wenckus CS. Effect of magnification on locating the MB2 canal in maxillary molars. J Endod. 2002; 28(4):324-7.

11. Pardo MCP, Nishiyama CK, Ponce JB, Albarracín ML. CBCT and microscopic analysis of the incidence of second mesiobuccal canal of maxillary molars. Dental Press Endod. 2012; 2(3):61-6.

12. Hartmann, MS, Ferreira P, Baratto Filho F, Fariniuk LF, Limongi O, Pizzatto E. Clinical and microscopic analysis of the incidence of a fourth canal and its trajectory in the maxillary first molar. Rev Gaúch Odontol. 2009; 57(4):381-4.

13. Neelakantan P, Subbarao C, Subbarao CV. Comparative evaluation of modified canal staining and clearing technique, CBCT, peripheral quantitative computed tomography, spiral computed tomography, and plain and contrast medium-enhanced digital radiography in studying root canal morphology. J Endod. 2010; 36(9):1547-51.

14. Alacam T, Tinaz AC, Genc O, Kayaoglu G. Second mesiobuccal canal detection in maxillary first molars using microscopy and ultrasonics. Aus Endod J. 2008; 34(3):106-9.

15. Vertucci FJ. Root canal anatomy of the human permanent teeth. Oral Surg Oral Med Oral Pathol. 1984; 58(5):589-99.

16. Faramarzi F, Vossoghi M, Sokri A, Shams B, Khoshbin E. Cone Beam Computed Tomography study of root and canal morphology of maxillary first molar in an Iranian population. Avicenna J Dent Res. 2015;7(1):e24038

17. Rwenyonyi CM, Kutesa AM, Muwazi M, Buwembo W. Root and canal morphology of maxillary first and second permanent molar teeth in a Ugandan population. Int Endo J. 2007; 40: 679-83.

18. Weine FS, Hayami S, Hata G, Toda T. Canal configuration of the mesiobuccal root of the maxillary first molar of a Japanese subpopulation. Int Endo J.1999; 32:78-9.

19. Zhang CF, Ding RY, Yin XZ, Zhao BH, Lin QG. Location and negotiation of second mesiobuccal canals in maxillary molars. Zhonghua KouQiang YiXueZaZhi. 2003; 38(2):86-8.

20. Das S, Warhadpande MM, Redij SA, Jibhkate NG, Sabir H. Frequency of second mesiobuccal canal in permanent maxillary first molars using the operating microscope \& selective dentin removal: A clinical study. Contemp Clin Dent. 2015; 6(1):74-8. 